\title{
Disagreement in primary study selection between systematic reviews on negative pressure wound therapy Frank Peinemann*1, Natalie McGauran ${ }^{1}$, Stefan Sauerland ${ }^{2}$ and Stefan Lange ${ }^{1}$
}

\author{
Address: ${ }^{1}$ Institute for Quality and Efficiency in Health Care (IQWiG), Dillenburger Str. 27, 51105 Cologne, Germany and ${ }^{2}$ Institute for Research \\ in Operative Medicine, University of Witten/Herdecke, Ostmerheimer Str. 200, 51109 Cologne, Germany \\ Email: Frank Peinemann* - frank.peinemann@iqwig.de; Natalie McGauran -n.mcgauran@iqwig.de; Stefan Sauerland - stefan.sauerland@ifom- \\ uni-wh.de; Stefan Lange - stefan.lange@iqwig.de \\ * Corresponding author
}

Published: 26 June 2008

BMC Medical Research Methodology 2008, 8:4I doi:10.1186/I47|-2288-8-4I

This article is available from: http://www.biomedcentral.com/|47|-2288/8/4I

(c) 2008 Peinemann et al; licensee BioMed Central Ltd.

This is an Open Access article distributed under the terms of the Creative Commons Attribution License (http://creativecommons.org/licenses/by/2.0), which permits unrestricted use, distribution, and reproduction in any medium, provided the original work is properly cited.
Received: 7 January 2008

Accepted: 26 June 2008

\begin{abstract}
Background: Primary study selection between systematic reviews is inconsistent, and reviews on the same topic may reach different conclusions. Our main objective was to compare systematic reviews on negative pressure wound therapy (NPWT) regarding their agreement in primary study selection.

Methods: This retrospective analysis was conducted within the framework of a systematic review (a full review and a subsequent rapid report) on NPWT prepared by the Institute for Quality and Efficiency in Health Care (IQWiG).

For the IQWiG review and rapid report, 4 bibliographic databases (MEDLINE, EMBASE, The Cochrane Library, and CINAHL) were searched to identify systematic reviews and primary studies on NPWT versus conventional wound therapy in patients with acute or chronic wounds. All databases were searched from inception to December 2006.

For the present analysis, reviews on NPWT were classified as eligible systematic reviews if multiple sources were systematically searched and the search strategy was documented. To ensure comparability between reviews, only reviews published in or after December 2004 and only studies published before June 2004 were considered.
\end{abstract}

Eligible reviews were compared in respect of the methodology applied and the selection of primary studies.

Results: A total of 5 systematic reviews (including the IQWiG review) and 16 primary studies were analysed. The reviews included between 4 and 13 primary studies published before June 2004. Two reviews considered only randomised controlled trials (RCTs). Three reviews considered both RCTs and non-RCTs. The overall agreement in study selection between reviews was $96 \%$ for RCTs ( 24 of 25 options) and $57 \%$ for non-RCTs (I 2 of 21 options). Due to considerable disagreement in the citation and selection of non-RCTs, we contacted the review authors for clarification (this was not initially planned); all authors or institutions responded. According to published information and the additional information provided, most differences between reviews arose from variations in inclusion criteria or interauthor study classification, as well as from different reporting styles (citation or non-citation) for excluded studies.

Conclusion: The citation and selection of primary studies differ between systematic reviews on NPWT, particularly with regard to non-RCTs. Uniform methodological and reporting standards need to be applied to ensure comparability between reviews as well as the validity of their conclusions. 


\section{Background}

Although systematic reviews are a valuable tool in the synthesis of evidence, they should be interpreted with caution [1]. The sharp rise in the number of systematic reviews published over the past decades has led to a concomitant increase in discordant results and conclusions between reviews on the same research question [2-5]. This has caused disputes between researchers and created difficulties for decision-makers in selecting appropriate health care interventions. Among other things, discordance between reviews may be caused by differences in primary study selection [6] due to variations in literature search strategies, selection criteria, and the application of selection criteria [2].

The Institute for Quality and Efficiency in Health Care (Institut für Qualität und Wirtschaftlichkeit im Gesundheitswesen, IQWiG) conducted a systematic review on the effectiveness and safety of negative pressure wound therapy (NPWT) versus conventional wound therapy in patients with acute or chronic wounds. The NPWT technique aims to accelerate wound healing by placing a foam dressing in the wound and applying controlled subatmospheric pressure [7]. The German-language full review and a rapid report on studies subsequently published are available on the IQWiG website [8,9]. In addition, an English-language journal article has been published [10].

An additional retrospective analysis was conducted in order to compare different systematic reviews on NPWT regarding their agreement in primary study selection. The review methodologies were also compared.

\section{Methods}

For the IQWiG review and rapid report, 4 bibliographic databases (MEDLINE, EMBASE, The Cochrane Library, and CINAHL) were searched to identify systematic reviews and primary studies on NPWT versus conventional wound therapy in patients with acute or chronic wounds. All databases were searched from inception to May 2005 (review) and between May 2005 and December 2006 (rapid report).

The multi-source search strategy and literature screening are described in detail elsewhere [8]. Eligible primary studies were randomised controlled trials (RCTs), as well as non-randomised controlled trials (non-RCTs) with a concurrent control group. Studies were classified as nonrandomised if allocation concealment was viewed as inadequate [11]. Quasi-randomised studies were therefore classified as non-randomised. The intervention was categorised as NPWT if a medical device system identical or comparable to the vacuum-assisted closure (V.A.C. $\left.{ }^{\oplus}\right)$ system was used. Studies were considered to be eligible only if publicly accessible full-text articles or other com- prehensive study information (e.g. clinical study reports provided by manufacturers) were available.

For the present analysis, an identical and sufficiently large primary study pool, i.e. the pool of studies that could potentially be identified by all reviews, was required to ensure comparability between reviews. As a preliminary analysis showed that early reviews merely included 2 to 4 primary studies, only reviews published in or after December 2004 were considered.

Eligible reviews had to include data from completed primary studies on NPWT. Reviews were classified as systematic reviews (as opposed to narrative reviews) if multiple sources were searched (at least MEDLINE and The Cochrane Library), and the search strategy (including the search date) was documented [12].

Primary studies were eligible for inclusion only if they had been published before June 2004 and if the entry date of a study in a database preceded the date of the literature search of any systematic review analysed.

The methodology and primary study selection between reviews were compared, and the overall agreement in study selection between reviews was reported.

Only a summary of the reviews' quality assessment of primary studies and their conclusions on the effectiveness of NPWT is presented here, as the main focus of this paper was to compare the agreement in primary study selection between reviews.

\section{Results}

The flow charts of the selection of systematic reviews and primary studies are presented in Figures 1 and 2. Sixteen primary studies published before June 2004 were assessed in the present analysis [13-28]. A total of 5 eligible systematic reviews (the IQWiG review and 4 other systematic reviews) published between December 2004 and July 2006 were analysed [29-32]. Details on all reviews identified are shown in Table 1; the main reason for exclusion was failure to qualify as a systematic review.

The methods applied in the reviews included are presented in Tables 2 and 3. Regarding bibliographic databases, all reviews used MEDLINE, EMBASE, and The Cochrane Library, but the nursing database CINAHL was used only by IQWiG. The search terms applied varied between reviews. Regarding study design, the IQWiG review [8], as well as the reviews by Costa 2005 [30] and Pham 2006 [31] considered both RCTs and non-RCTs, while the reviews by Samson 2004 [29] and OHTAC 2006 [32] took only RCTs into account. 
Table I: Identified pool of potentially relevant reviews

\begin{tabular}{|c|c|c|c|c|c|}
\hline No & Publication & Documentation* & Multiple sources ${ }^{\dagger}$ & Included $\ddagger$ & Reason for exclusion \\
\hline 1 & Andros 2006 [53] & - & - & - & Consensus statement \\
\hline 2 & Brem 2006 [54] & - & - & - & Guidelines \\
\hline 3 & Costa/MUHC TAU $2005[30]^{\S}$ & + & + & + & - \\
\hline 4 & Evans 200I [55] & + & + & - & Systematic review published before 12/2004 \\
\hline 5 & Fleck 2006 [56] & - & - & - & Consensus statement \\
\hline 6 & Fisher 2003 [57] & - & - & - & Narrative review \\
\hline 7 & Gray 2004 [58] & + & + & - & Systematic review published before 12/2004 \\
\hline 8 & Hayes Inc. 2003 [59] & & & - & $\begin{array}{l}\text { Not publicly accessible; published before I2/ } \\
2004\end{array}$ \\
\hline 9 & Higgins 2003 [60] & + & + & - & Systematic review published before 12/2004 \\
\hline 10 & Mayer 2002 [6I] & + & + & - & $\begin{array}{l}\text { Not publicly accessible; published before I2/ } \\
2004\end{array}$ \\
\hline 11 & Mendonca 2006 [62] & - & + & - & Search date not reported \\
\hline 12 & OHTAC 2004 [63] & + & + & - & Update available: OHTAC 2006 \\
\hline 13 & OHTAC 2006 update [32] & + & + & + & - \\
\hline 14 & Pham/ASERNIP-S 2003 [64] & + & + & - & Update available: Pham 2006 \\
\hline 15 & Pham/ASERNIP-S 2006 update [3I] & + & + & + & - \\
\hline 16 & Samson/AHRQ 2004 [29] & + & + & + & - \\
\hline 17 & Shirakawa 2005 [65] & - & - & - & Narrative review \\
\hline 18 & Suess 2006 [66] & - & - & - & Narrative review \\
\hline 19 & Turina 2006 [67] & - & - & - & Narrative review \\
\hline 20 & Ubbink 2006 [68] & - & - & - & Narrative review \\
\hline 21 & Whelan 2005 [69] & - & - & - & Article on mechanisms of wound healing \\
\hline
\end{tabular}

*The literature search strategy was documented in detail (including search strategy and search date). tMultiple sources used (at least MEDLINE and The Cochrane Library).

$\ddagger$ Criteria fulfilled: detailed documentation of the literature search; use of multiple sources; publication in or after December 2004.

§osta 2007 [70] was not classified as an update, as no update of the literature search was performed.

AHRQ: Agency for Healthcare Research and Quality; ASERNIP-S: Australian Safety and Efficacy Register of New Interventional Procedures -

Surgical; IQWiG: Institut für Qualität und Wirtschaftlichkeit im Gesundheitswesen (Institute for Quality and Efficiency in Health Care); MUHC

TAU: McGill University Health Centre Technology Assessment Unit; NPWT: negative pressure wound therapy; OHTAC: Ontario Health

Technology Advisory Committee.

As the comparison of systematic reviews based on published information showed numerous inconsistencies, we decided to contact the authors of the other reviews for clarification (this was not initially planned). We received responses from all authors approached (or from other researchers at the publishing institutions). After reviewing the responses, it became clear that reporting styles for excluded studies differed between reviews. For example, the response by OHTAC stated that "it must be noted that we do not routinely cite or analyse studies that have been excluded from our EBAs (evidence-based analyses)" [personal communication]. It consequently became apparent that some studies we had initially classified as "not identified by other reviews" had actually been identified but excluded, and subsequently not reported. We therefore changed the classification of studies not cited in reviews to "not reported". In addition, the authors of reviews corrected or clarified published information (their comments are included in Tables 4, 5, 6); in this context we thank them for generously providing information.

Details of the primary study selection are presented according to the study classification by IQWiG in Tables 4
(5 RCTs), 5 (7 non-RCTs), and 6 (3 non-RCTs and 1 RCT excluded by IQWiG, but included by at least one other review).

The reviews included between 4 and 13 eligible primary studies published before June 2004. With regard to RCTs, the overall agreement in primary study selection between reviews was $96 \%$ (24 of 25 options) (Table 5).

More variations were noted concerning the selection of non-RCTs; the agreement between reviews considering both RCTs and non-RCTs was 57\% (12 of 21 options). Of the 9 mismatches, according to published information and the information provided by authors or institutions, 7 were due to different inclusion criteria (e.g. language criteria), and 2 were due to variations in study classification (Table 5).

Four studies ( 3 non-RCTs and 1 RCT) were excluded by IQWiG but included by at least one other review. The reasons for exclusion were as follows: the study included historical controls (2 non-RCTs $[13,26])$; the intervention applied was not comparable to the NPWT technique (1 

(search in May 2005)

Included in IQWiG's rapid report (search in December 2006)

Inclusion: Internet (search in December 2006)

Potentially relevant for the analysis

Exclusion: Not regarded to fulfill criteria for a systematic review

Exclusion: Systematic reviews issued in or before December 2004

Exclusion: Review updates available

Relevant for the analysis
Included in IQWiG's final report

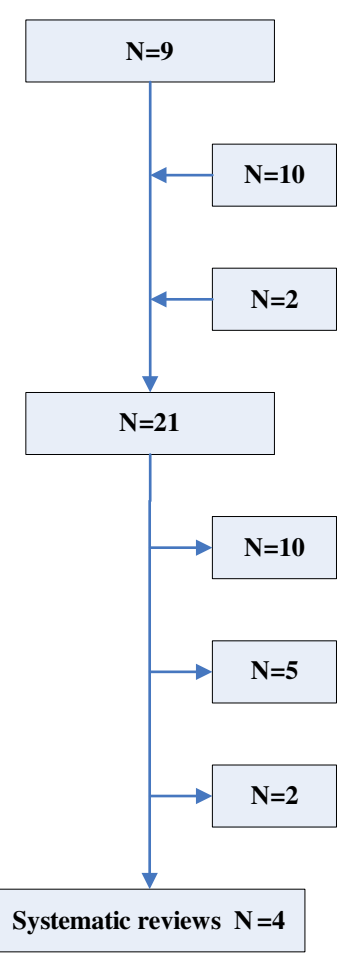

Figure I

Flow chart of the review selection.

non-RCT [14]); or an additional intervention was applied that may have affected the study outcomes (1 RCT [19]) (Table 6). Substantial variations in study selection were shown between reviews.

Included in IQWiG's final report (search in May 2005)

Included in IQWiG's rapid report (search in December 2006)

Included in at least one of the 4 other identified systematic reviews

Potentially relevant for the analysis

Exclusion: Studies entered into the literature databases in or after June 2004

Relevant for the analysis

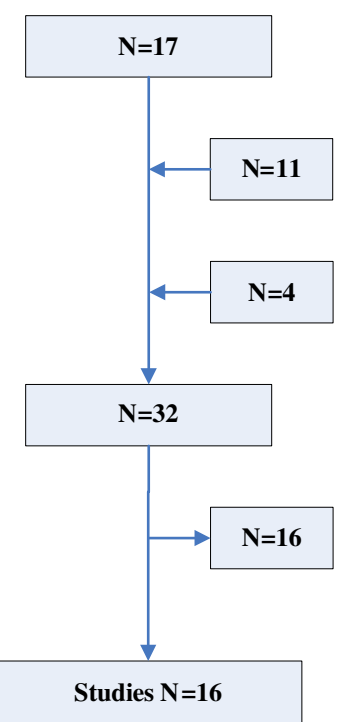

Figure 2

Flow chart of the study selection.
Only the IQWiG review included a meta-analysis (changes in wound size), which indicated an advantage in favour of NPWT. However, only a few trials with small sample sizes were analysed.

The overall quality of the primary studies was assessed in 3 of 5 reviews, and was in general classified as poor. All reviews concluded that the evidence base on NPWT was insufficient (Table 7).

\section{Discussion}

An analysis of 5 systematic reviews on NPWT showed differences (which mainly concerned non-RCTs) in the citation and selection of primary studies.

We would like to emphasize that by presenting these differences, we are not implying that the 4 other reviews identified were of inferior quality compared with the IQWiG review. Variations in the number of primary studies identified and selected are not surprising, as the reviews used different search strategies, literature sources, and inclusion criteria. After correspondence with the authors of the other reviews, many differences regarding the citation of primary studies could be attributed to different reporting styles (citation or non-citation) for excluded studies, not to the non-detection of studies in the literature searches.

Most differences in study selection resulted from variations in inclusion and exclusion criteria. For example, due to language restrictions, studies published in German were selected by IQWiG, but not by other reviews. Opinions on the relevance of language bias differ; a study published in 1997 comparing English and German-language publications concluded that English-language bias may be introduced in systematic reviews if they include only trials reported in English [33]. In contrast, a more recent publication noted that, for conventional medicinal interventions, language restrictions did not appear to bias estimates of effectiveness [34]. Moreover, for Germanlanguage publications on RCTs, it has been reported that German medical journals no longer play a role in the dissemination of trial results [35].

The inclusion criteria for primary study design were also inconsistent; 3 reviews (including the IQWiG review) considered both RCTs and non-RCTs, and 2 reviews considered only RCTs. The non-RCTs included in our analysis were non-randomised controlled intervention studies. However, there are many different study types that can be seen as non-RCTs (e.g., classical observational studies). The inclusion of non-RCTs in systematic reviews is inconsistent and controversial [36-40]. The validity of systematic reviews including non-RCTs may be affected by the differing susceptibility of RCTs and non-RCTs to selection 
Table 2: Systematic reviews on NPWT: Requirements for primary studies and publications

\begin{tabular}{|c|c|c|c|c|c|c|c|c|}
\hline Systematic review & Interventions & Wound types & Clinical outcomes & $\begin{array}{l}\text { Clinical study } \\
\text { design }\end{array}$ & $\begin{array}{l}\text { Sample size } \\
\text { restrictions }\end{array}$ & $\begin{array}{l}\text { Type of study } \\
\text { information } \\
\text { considered }\end{array}$ & $\begin{array}{l}\text { Prespecified } \\
\text { search for HTAs/ } \\
\text { SRs }\end{array}$ & $\begin{array}{l}\text { Language } \\
\text { restrictions }\end{array}$ \\
\hline $\begin{array}{l}\text { Samson/AHRQ } \\
2004 \text { [29] }\end{array}$ & $\begin{array}{l}\text { a) NPWT vs. other } \\
\text { wound healing } \\
\text { interventions } \\
\text { b) NPWT + standard } \\
\text { care vs. standard } \\
\text { wound care alone } \\
\text { c) NPWT vs. sham } \\
\text { intervention }\end{array}$ & $\begin{array}{l}\text { Chronic wounds: } \\
\text { - pressure ulcers } \\
\text { - metabolic } \\
\text { disorders (e.g., } \\
\text { diabetes mellitus) } \\
\text { - vascular } \\
\text { insufficiency } \\
\text { - inflammatory } \\
\text { disorders } \\
\text { - malignancies } \\
\text { - infections } \\
\text { - miscellaneous (e.g., } \\
\text { burns) } \\
\text { Other types of } \\
\text { wounds: } \\
\text { - acute wounds } \\
\text { - traumatic wounds } \\
\text { - subacute wounds } \\
\text { - dehisced wounds } \\
\text { - partial thickness } \\
\text { burns } \\
\text { - diabetic ulcers } \\
\text { - pressure ulcers } \\
\text { - flaps } \\
\text { - grafts }\end{array}$ & $\begin{array}{l}\text { Primary outcomes: } \\
\text { - incidence of } \\
\text { complete wound } \\
\text { closure } \\
\text { - time to complete } \\
\text { closure } \\
\text { - adverse events } \\
\text { Secondary outcomes: } \\
\text { - facilitating surgical } \\
\text { closure } \\
\text { - need for } \\
\text { debridement } \\
\text { - infections } \\
\text { - pain } \\
\text { - activities of daily } \\
\text { living } \\
\text { - quality of life } \\
\text { - improved cosmesis } \\
\text { - change in wound } \\
\text { size* } \\
\text { - transcutaneous } \\
\text { oxygen tension* }\end{array}$ & RCT & None & Articles & - & $\begin{array}{l}\text { Abstracts published } \\
\text { in English; articles } \\
\text { without abstracts } \\
\text { were reviewed if } \\
\text { title indicated that } \\
\text { articles met inclusion } \\
\text { criteria; non-English } \\
\text { articles were } \\
\text { reviewed if English } \\
\text { abstract indicated } \\
\text { that articles met } \\
\text { inclusion criteria. }\end{array}$ \\
\hline $\begin{array}{l}\text { Costa/MUHC } \\
\text { TAU } 2005[30]^{\dagger}\end{array}$ & $\begin{array}{l}\text { NPWT vs. other } \\
\text { treatment } \\
\text { alternatives }\end{array}$ & $\begin{array}{l}\text { Not prespecified in } \\
\text { detail }\end{array}$ & $\begin{array}{l}\text { Not prespecified in } \\
\text { detail ("clinical } \\
\text { effectiveness") }\end{array}$ & $\begin{array}{l}\text { RCT } \\
\text { Non-RCT (clinical } \\
\text { comparative studies) }\end{array}$ & $\begin{array}{l}\geq 9 \text { patients in either } \\
\operatorname{arm}^{\ddagger}\end{array}$ & Articles & + & $\begin{array}{l}\text { Articles published in } \\
\text { English or French }\end{array}$ \\
\hline IQWiG 2006 [8] & $\begin{array}{l}\text { NPWT vs. } \\
\text { a) conventional } \\
\text { wound therapy } \\
\text { b) another type of } \\
\text { NPWT }\end{array}$ & $\begin{array}{l}\text { Acute or chronic } \\
\text { wounds }\end{array}$ & $\begin{array}{l}\text { - wound healing time } \\
\text { - wound recurrence } \\
\text { - revision operations } \\
\text { - amputations } \\
\text { - mortality } \\
\text { - disease-related } \\
\text { quality of life } \\
\text { - activities of } \\
\text { everyday life } \\
\text { - pain } \\
\text { - time spent in } \\
\text { hospital } \\
\text { - dressing changes } \\
\text { - debridement } \\
\text { procedures } \\
\text { - adverse events } \\
\text { - scar formation } \\
\text { - subjective cosmetic } \\
\text { results }\end{array}$ & $\begin{array}{l}\text { RCT } \\
\text { Non-RCT with a } \\
\text { concurrent control } \\
\text { group (clinical } \\
\text { controlled trials, } \\
\text { comparative cohort } \\
\text { studies, case control } \\
\text { studies) }\end{array}$ & None & $\begin{array}{l}\text { Articles } \\
\text { Unpublished data } \\
\text { provided by } \\
\text { manufacturers } \S\end{array}$ & + & $\begin{array}{l}\text { Language restrictions } \\
\text { were not specified in } \\
\text { the IQWiG review.II }\end{array}$ \\
\hline
\end{tabular}


Table 2: Systematic reviews on NPWT: Requirements for primary studies and publications (Continued)

\begin{tabular}{|c|c|c|c|c|c|c|c|c|}
\hline $\begin{array}{l}\text { Pham/ASERNI P- } \\
\text { S } 2006 \text { update } \\
{[31]^{\uparrow \mid}}\end{array}$ & $\begin{array}{l}\text { NPWT vs. } \\
\text { conventional } \\
\text { methods }\end{array}$ & $\begin{array}{l}\text { Particular wound } \\
\text { types** } \\
\text { - pressure ulcers and } \\
\text { leg ulcers } \\
\text { - diabetic foot ulcers } \\
\text { and wounds } \\
\text { - skin grafts } \\
\text { - chronic wounds } \\
\text { and complex/severe } \\
\text { wounds } \\
\text { - sternal wounds }\end{array}$ & $\begin{array}{l}\text { Not prespecified in } \\
\text { detail ("efficacy and } \\
\text { safety outcomes") }\end{array}$ & $\begin{array}{l}\text { RCT } \\
\text { Non-RCT (other } \\
\text { controlled or } \\
\text { comparative studies } \\
\text { and case series with } \\
\text { consecutive patients) }\end{array}$ & None & $\begin{array}{l}\text { Articles } \\
\text { Conference } \\
\text { abstractstt } \\
\text { Manufacturer's } \\
\text { informationtt }\end{array}$ & - & $\begin{array}{l}\text { Searches were } \\
\text { conducted without } \\
\text { language restriction. } \\
\text { English abstracts } \\
\text { from non-English } \\
\text { articles were } \\
\text { included if they met } \\
\text { the inclusion criteria } \\
\text { and included efficacy } \\
\text { and safety data. } \neq\end{array}$ \\
\hline $\begin{array}{l}\text { OHTAC } 2006 \\
\text { (update) }[32]^{\S \S}\end{array}$ & $\begin{array}{l}\text { NPWT vs. standard } \\
\text { care }\end{array}$ & $\begin{array}{l}\text { Wounds, including } \\
\text { - Pressure ulcers } \\
\text { - diabetic ulcers } \\
\text { - sternal wounds } \\
\text { - skin grafts }\end{array}$ & $\begin{array}{l}\text { Not prespecified in } \\
\text { detail ("Is negative } \\
\text { pressure wound } \\
\text { therapy effective for } \\
\text { healing wounds...?") }\end{array}$ & RCT & $\geq 20$ patients & $\begin{array}{l}\text { Articles } \\
\text { ("peer-reviewed, } \\
\text { published") }\end{array}$ & + & $\begin{array}{l}\text { Articles published in } \\
\text { English }\end{array}$ \\
\hline
\end{tabular}

*"Considered to be of less clinical importance" [29]

tCosta also considered economic outcomes.

$\ddagger$ One crossover study involving 7 patients was also included.

§Unpublished data from primary studies were only to be considered in the review if comprehensive study information (e.g. a clinical study report) was available.

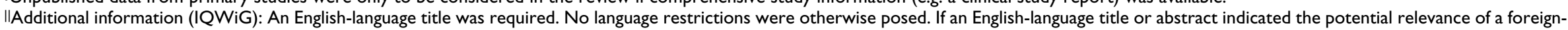
language text, the text was obtained and translated.

TPersonal communication (C. Perera, ASERNIP-S): "This publication draws from an accelerated systematic review which was published in 2003 and is accessible at http://www.surgeons.org/AM/

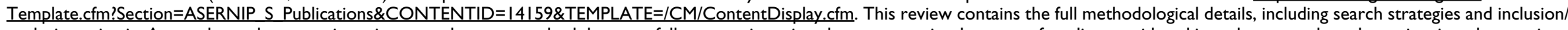
作 period than the full systematic review. For example, accelerated reviews generally only include comparative studies and not case series, unless safety outcomes were inadequately described in the

$\varnothing^{\infty} \quad$ comparative evidence."

N t+"Conference abstracts and manufacturer's information were included if they contained relevant safety and efficacy data." [3।]

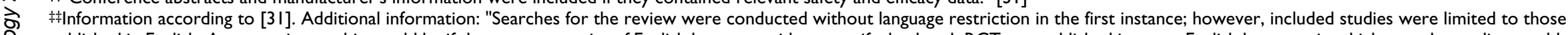

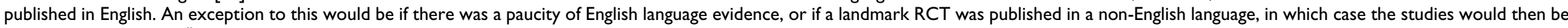
translated and included." (Personal communication: C. Perera, ASERNIP-S).

$\$ \$ O H T A C$ also considered economic outcomes.

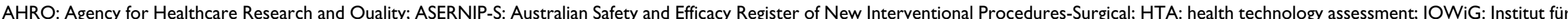
Qualität und Wirtschaftlichkeit im Gesundheitswesen (Institute for Quality and Efficiency in Health Care); MUHC TAU: McGill University Health Centre Technology Assessment Unit; NPWT: negative pressure wound therapy; OHTAC: Ontario Health Technology Advisory Committee; RCT: randomised controlled trial; SR: systematic review. 
$\mp$ Table 3: Systematic reviews on NPWT: Search strategies

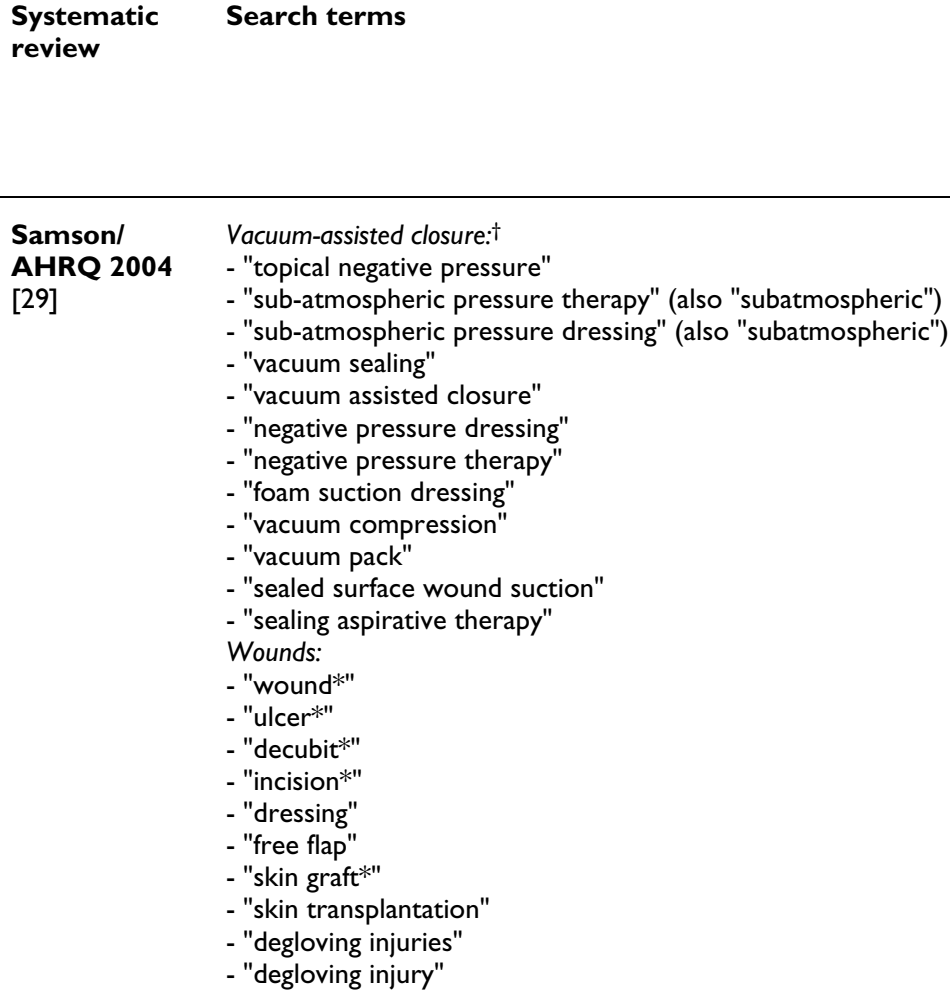

+
PubMed

+
+
Ovid MEDLINE,
Ovid MEDLINE
In-Process and
Other Non-
Indexed Citations

Literature sources

Search date*

MEDLINE

EMBASE

The Cochrane

CINAHL

Others

Library

MEDLINE via

$+$

PubMed

$+$

CENTRAL

-

$6 / 20045$

Manufacturers $\ddagger$

C

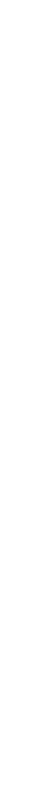

HTA agencies (CHSPR,

MCHP, ICES, INAHTA)

- Screening of reference

lists of primary studies and

secondary publications

$\begin{array}{cccl}+ & + & + & - \\ \text { Ovid EMBASE } & \text { CENTRAL; } & \text { Ovid CINAHL } & \text { Trial registries } \\ & \text { CDSR; DARE; } & & \text { (ClinicalTrials.gov, } \\ & \text { HTA } & \text { National Research } \\ & & \text { Register) } \\ & & - \text { Manufacturerst } \\ & & - \text { Authorities } \\ & & - \text { Conference proceedings } \\ & & - \text { Authors of articles and } \\ & & \text { conference abstracts } \\ & & - \text { Screening of reference } \\ & & \text { lists of secondary } \\ & \text { publications }\end{array}$

$3 / 2005$

$5 / 2005$ 
Pham/ (vacuum or suction) and (wound healing), (vacuum assisted or ASERNIP-S vacuum-assisted) and (wound or closure), topical negative 2006 update pressure, (subatmospheric or sub-atmospheric) and pressure [3I]
MEDLINE; PREMEDLINE PubMed

+
The Cochrane
Library

Current Contents

- Trial registries

(ClinicalTrials.gov,

National Research

Register)

- The York (UK) Centre

for Reviews and

Dissemination

- Grey literature reports

- Relevant online journals

- Vacuum therapy website

(vacuumtherapy.co.uk)

- The Internet

\section{OHTAC 2006 .}

(update) [32] wound healing

- foot ulcer or skin ulcer or varicose ulcer or leg ulcer

- wounds, non penetrating

- chronic and ulcer or wound

- leg or foot arterial or diabetic and ulcer or wound

- suction

- pressure

- vacuum

- vacuum assisted closure or V.A.C. therapy

- negative pressure

- topical negative pressure

$\underset{+}{\dot{\infty}}$

- subatmospheric pressure therapy

$\varnothing^{\infty}$ *End of search period.

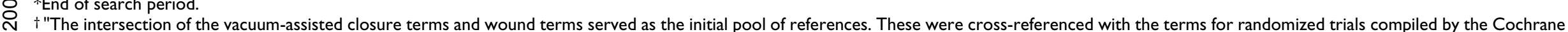

Collaboration...." For further details, please see Appendix A [29].

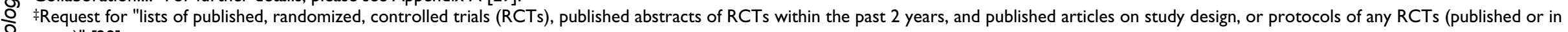
press)" [29].

§CENTRAL: 2003 ("through issue number 4", [29]).

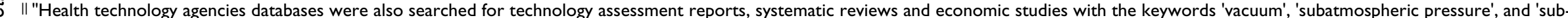
atmospheric pressure' used individually" [30].

TUnpublished data from primary studies were only to be considered in the review if comprehensive study information (e.g. a clinical study report) was available.

** "Updated searches were performed in July 2005 to include any new RCTs" [3I]

AHRQ: Agency for Healthcare Research and Quality; ASERNIP-S: Australian Safety and Efficacy Register of New Interventional Procedures - Surgical; CDSR: Cochrane Database of Systematic Reviews;

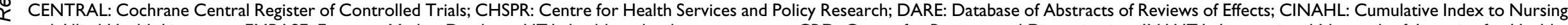

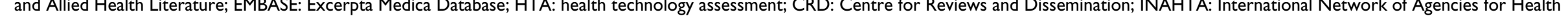

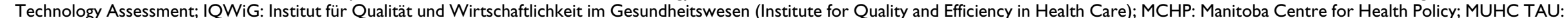

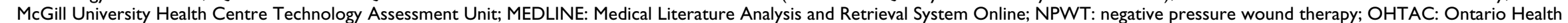

$\sum$ Technology Advisory Committee; RCT: randomised controlled trial; VAC: vacuum-assisted closure. 
Table 4: Overview of primary study selection: comparison of trials included as RCTs by IQWiG

\begin{tabular}{|c|c|c|c|}
\hline Systematic review & Search date & Primary study* (database entry date) ${ }^{\dagger}$ & $\begin{array}{l}\text { Personal } \\
\text { communication with } \\
\text { review authors or other } \\
\text { researchers at the } \\
\text { publishing institutions }\end{array}$ \\
\hline
\end{tabular}

Joseph 2000 [20] Ford 2002 [17] Wanner 2003 [27] Eginton 2003 [16] Moues 2004 [23]

(CIN: 2000-08; C) (M: 2002-07-27; E; C) (M: 2003-03-II; E; C) (M: 2003-10-10; E; C) (M: 2004-02-2I; E; C;

\section{IQWiG 2006 [8]}

$5 / 2005$

RCT

RCT

RCT

RCT 2006 update/[3I] $\quad$ RCTs) 7/2005 (RCTs)

\section{Reviews}

considering RCTs

Samson/AHRQ

2004 [29]

OHTAC 2006 [32]

$6 / 2004$

$3 / 2006$
RCT

RCT
RCT
RCT

RCT

RCT

RCT
RCT

RCT

RCT

Not reported (PC:

excluded)
Eginton 2003. PC (V. Costa): "We classified the study as a crossover design. If I had to discern between RCT and non-RCT, I would consider it an RCT."

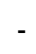

RCT

RCT

RCT

Eginton 2003. PC (Medical Advisory Secretariat): "This citation was retrieved from the literature search, but was excluded as only 10 patients were enrolled. The exclusion was not documented in the HTA.

*Unless otherwise noted, the language of publication (abstract and full text) is English.

tDatabases containing primary studies (entry date in database: yyyy-mm-dd).

‡"Crossover; first treatment selected randomly"[30].

AHRQ: Agency for Healthcare Research and Quality; ASERNIP-S: Australian Safety and Efficacy Register of New Interventional Procedures - Surgical; C: The Cochrane Library; CIN: CINAHL; E:

EMBASE; HTA: health technology assessment; IQWiG: Institut für Qualität und Wirtschaftlichkeit im Gesundheitswesen (Institute for Quality and Efficiency in Health Care); M: MEDLINE; MUHC TAU: McGill University Health Centre Technology Assessment Unit; OHTAC: Ontario Health Technology Advisory Committee; PC: personal communication; RCT: randomised controlled trial. 
$\subsetneq$ Table 5: Overview of primary study selection: comparison of trials included as non-RCTs by IQWiG

\begin{tabular}{|c|c|c|c|c|c|c|c|c|c|}
\hline \multirow{2}{*}{$\begin{array}{l}\text { Systematic } \\
\text { review }\end{array}$} & \multirow[t]{2}{*}{ Search date } & \multicolumn{7}{|c|}{ Primary study* (Database entry date) ${ }^{\dagger}$} & \multirow{2}{*}{$\begin{array}{l}\text { Personal communication with review authors } \\
\text { or other researchers at the publishing } \\
\text { institutions }\end{array}$} \\
\hline & & $\begin{array}{l}\text { Genecov } \\
1998[18]\end{array}$ & $\begin{array}{l}\text { McCallon } \\
2000[22]\end{array}$ & $\begin{array}{c}\text { Doss } 2002 \\
{[15]}\end{array}$ & $\begin{array}{l}\text { Scherer } \\
2002[24]\end{array}$ & $\begin{array}{l}\text { Kamolz } \\
2004 \text { [2I] }\end{array}$ & $\begin{array}{l}\text { Schrank } \\
2004[25]^{\ddagger}\end{array}$ & $\begin{array}{c}\text { Wild } 2004 \\
{[28]^{\ddagger}}\end{array}$ & \\
\hline & & $\begin{array}{c}\text { (M: 1998-04- } \\
02 ; \mathrm{E} ; \mathrm{C})\end{array}$ & $\begin{array}{l}\text { (M: 200I-02- } \\
24 ; C ; C I N)\end{array}$ & $\begin{array}{l}\text { (M: 2002-12- } \\
07 ; \mathrm{E} ; \mathrm{C})\end{array}$ & $\begin{array}{c}\text { (M: 2002-07- } \\
3 \mathrm{I} ; \mathrm{E})\end{array}$ & $\begin{array}{l}\text { (M: 2004-04- } \\
\text { I5; E; CIN) }\end{array}$ & $\begin{array}{c}\text { (M: 2004-05- } \\
\text { 29; E; C) }\end{array}$ & $\begin{array}{c}\text { (M: 2004-05- } \\
29 ; \mathrm{E})\end{array}$ & \\
\hline
\end{tabular}

considering

RCTs and

non-RCTs

Costal

MUHCTAU

2005 [30]

3/2005 Non-RCT

reason not

stated in

review (PC:

sample size

too small)

\begin{tabular}{|c|c|c|}
\hline $\begin{array}{l}\text { IQWiG } \\
2006 \text { [8] }\end{array}$ & $5 / 2005$ & Non-RCT \\
\hline
\end{tabular}

was based on

alterna-tion)

Non-RCT

Non-RCT

(PC:

excluded)

(not

Not reported

(not

applicable§)

Non-RCT

Non-RCT

dy
a clinical but a surrogate outcome. "The perfusion of
both hands was measured using the technique of dynamic laser-fluorescence-videography" [2I]. IQWiG included this study because the outcome "pain" was reported in the results section ("All patients tolerated the V.A.C. application without major reports of pain and discomfort" [2I]), although the method of pain measurement was unclear.

Not report

(PC:

excluded)
Not reported

(PC:

excluded)

Genecov 1998. PC (C. Perera): "Allocation not stated, assumed that a valid method of randomisation had been utilised (critical appraisal in the full accelerated systematic review drew attention to this)."

McCallon 2000. PC (C. Perera): "As McCallon et al described this study as an RCT, the authors of the systematic review chose to classify it in the same way, despite the fact that patients were randomised based on alteration."

Kamolz 2004. PC (C. Perera): "Regarding the Kamolz study, treatment with VAC was focused upon the period immediately following trauma and thus these were not considered to be chronic, non-healing wounds which were the focus of this review and the manuscript."

Schrank 2004; Wild 2004. PC (C. Perera): "Regarding the Wild and Schrank studies, these were not published in English, however you are correct that these studies were published within the search dates. Had these studies been landmark RCTs, they would have been translated and included in the review." 
Table 5: Overview of primary study selection: comparison of trials included as non-RCTs by IQWiG (Continued)

Excluded: no outcomes of

interest
(RCT)

\section{Excluded: <} 20 patients (RCT)

\section{Excluded: < 20 patients} (RCT)

Not reported
(not
applicablef)

ot reported
(not
applicableI)

Excluded, as the trial was a non-RCT (not applicable
Not reported

(not

applicableI)
Genecov 1998. PC (D. Samson): "This study was not a parallel groups or crossover randomized trial, but it was a within-subjects experimental design in which each participant served as his/her own control by receiving Opsite wound dressing and vacuum-assisted closure to separate wounds or wound areas. Since our review was focused on the primary outcome of review was focused on the primary outcome of
progress to full wound healing and this study addressed only biopsy findings, this trial was excluded for reporting a non-relevant outcome."

McCallon 2000. PC (D. Samson): "Rather than excluding a marginal study like this based on quality concerns, our review selected an inclusive pool of randomized controlled trials, then evaluated study quality, noting that this trial ...used an allocation method that was probably inadequate to be considered true randomization (p. 57)."

\begin{tabular}{|c|c|c|c|}
\hline $\begin{array}{c}\text { Not reported } \\
\text { (not } \\
\text { applicableף) }\end{array}$ & $\begin{array}{c}\text { Not reported } \\
\text { (not } \\
\text { applicableף) }\end{array}$ & $\begin{array}{c}\text { Not reported } \\
\text { (not } \\
\text { applicableI) }\end{array}$ & $\begin{array}{c}\text { Not reported } \\
\text { (not } \\
\text { applicableI) }\end{array}$ \\
\hline
\end{tabular}

Not reported (not

Genecov 1998. PC (Medical Advisory Secretariat): "...the Genecov study is a case series of ten subjects and was
incorrectly referred to as a randomized controlled trial incorrectly referred to as a randomized controlled trial
(RCT). The study was excluded so how it was classified is not of particular relevance."

McCallon 2000. PC (Medical Advisory Secretariat): ".. excluded based on the information reported in the abstract; there were less than 20 patients and the study was designated as an RCT by MEDLINE. We would not was designated as an RCT by MEDLINE. We would
have retrieved the full text to further examine the study to determine how the randomization process was conducted given its exclusion based on number of subjects.

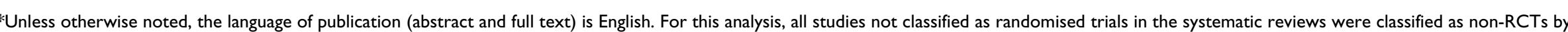
IQWiG.

tDatabases containing primary studies (entry date: yyyy-mm-dd).

‡German full text.

\$Review did not consider non-English or non-French full-text publications.

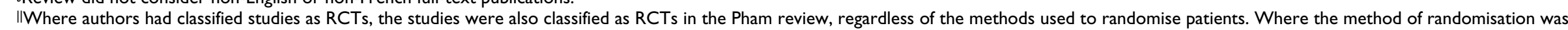
described, this was included in the critical appraisal section of the full systematic review, which is published on the ASERNIP-S website [personal communication: C. Perera, ASERNIP-S].

TReview did not consider non-RCTs.

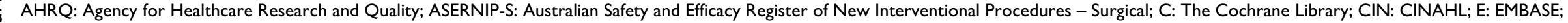

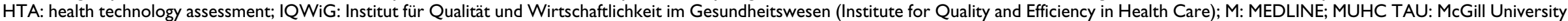
Health Centre Technology Assessment Unit; OHTAC: Ontario Health Technology Advisory Committee; PC: personal communications; RCT: randomised controlled trial. 
Table 6: Overview of primary study selection: comparison of trials excluded by IQWiG but included by at least one other review

\begin{tabular}{|c|c|c|c|c|c|c|}
\hline \multirow{2}{*}{$\begin{array}{l}\text { Systematic } \\
\text { review }\end{array}$} & \multirow[t]{2}{*}{ Search date } & \multicolumn{4}{|c|}{ Primary study* (Database entry date) ${ }^{\dagger}$} & \multirow{2}{*}{$\begin{array}{l}\text { Personal communication with review authors or other } \\
\text { researchers at the publishing institutions }\end{array}$} \\
\hline & & $\begin{array}{l}\text { Davydov } 1994 \\
{[14]^{\ddagger}} \\
\text { (M: 1994-09-0I; C) }\end{array}$ & $\begin{array}{l}\text { Catarino } 2000 \\
{[13]} \\
\text { (M: 200I-0I-I3) }\end{array}$ & $\begin{array}{l}\text { Song } 2003 \text { [26] } \\
(M: 2002-12-24 ; E)\end{array}$ & $\begin{array}{l}\text { Jeschke } 2004 \text { [19] } \\
\text { (M: 2004-02-06; E; } \\
\text { C) }\end{array}$ & \\
\hline
\end{tabular}

\section{Reviews \\ considering RCTs}

and non-RCTs

Costa/MUHC

TAU 2005 [30]

$3 / 2005$

Not reported (not

applicable§)

Non-RCT

Jeschke 2004. PC (V. Costa): "Considered not eligible. Although negative pressure was used in one group, we considered that the main intervention studied was the Integra grafting and not vacuumassisted closure. Moreover, since the group using VAC was a combined intervention (Integra + VAC), we were not sure if this would influence the results making it not an adequate estimate of the results with VAC alone. For these reasons the study was considered ineligible."

\section{IQWiG $2006[8]$}

$5 / 2005$

Excluded: not

Excluded: historical

NPWT (non-RCT) control (non-RCT)

Excluded: historical control (non-RCT)

Excluded: outcomes in the test group

possibly affected by the additional intervention (RCT)

\section{Pham/ASERNIP-}

S 2006 updatel

[3I ]
10/2004 (RCTs/non- RCT RCTs) 7/2005

(RCTs)
Non-RCT

Non-RCT
Davydov 1994. A translation of the full text of this Russianlanguage article showed that the intervention was not a technique comparable to NPWT. Consequently, IQWiG did not include this study. No reference to a randomised allocation was found. Jeschke 2004. NPWT was applied in combination with fibrin glue-anchored Integra in the test group receiving NPWT, but not in the control group receiving standard therapy; therefore the outcomes in the test group may have been affected by the additional intervention. Consequently, IQWiG did not include this study

Davydov 1994. PC (C. Perera): "Allocation not stated, assumed that a valid method of randomisation had been utilised (critical appraisal in the full accelerated systematic review drew attention to this)"

Jeschke 2004. PC (C. Perera): "Agree with IQWiG comments regarding additional intervention. Suggest that this RCT was included due to a paucity of RCT evidence on this indication (skin grafts), and as the other included RCT for skin grafts had only a 7 day follow up." 


\section{Reviews}

considering only

$6 / 2004$

Not reported

(not applicable**)

Not reported

(not applicable**)

Not reported

(not applicable**)

Not reported

(PC: not identified)

Jeschke 2004. PC (D. Samson): "This study did not appear in our literature search, probably because it was not yet entered onto an electronic database by the date of our last search update."

\section{OHTAC [32] \\ (3/2006; RCT)}

Not reported (not applicable**)

\section{Not reported} (not applicable**)
Not reported (not applicable*
Not reported (PC: excluded)
Jeschke 2004. PC (Medical Advisory Secretariat): "...was included in the literature search results, but there were only twelve patients in the study so it was excluded. The exclusion was not documented in the HTA.

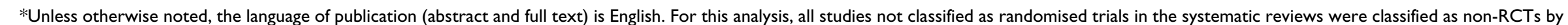
IQWiG. Studies not selected by any review are not listed in this table.

tDatabases containing primary studies (entry date: yyyy-mm-dd).

¥Russian full text.

\$Review did not consider non-English or non-French full-text publications.

IIStudy type not stated in the IQWiG review.

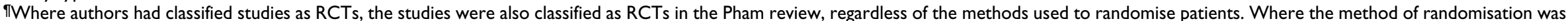
described, this was included in the critical appraisal section of the full systematic review, which is published on the ASERNIP-S website [Personal communication: C. Perera, ASERNIP-S] **Review did not consider non-RCTs.

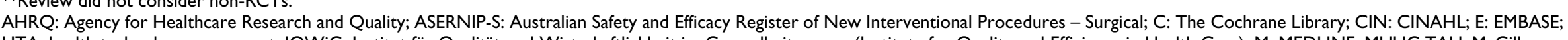
HTA: health technology assessment; IQWiG: Institut für Qualität und Wirtschaftlichkeit im Gesundheitswesen (Institute for Quality and Efficiency in Health Care); M: MEDLINE; MUHC TAU: McGill University Health Centre Technology Assessment Unit; NPWT: negative pressure wound therapy; OHTAC: Ontario Health Technology Advisory Committee; PC: personal communication; RCT:

randomised controlled trial; VAC: vacuum-assisted closure.

$\subsetneq$ Table 7: Overall quality assessment of primary studies and main conclusions of systematic reviews on negative pressure wound therapy

Systematic review Quality assessment conducted: yes/no and outcome* Main conclusion (direct quote)

के Samson/AHRQ 2004 [29] $\quad$ Yes. 6× poor in quality

\section{Costa/MUHC TAU 2005/[30]}

IQWiG [8]

Yes. $17 \times$ poor in quality

Pham/ASERNIP-S 2006 update [3I]

OHTAC [32]
No

Yes. I $\times$ moderate; $3 \times$ low; $2 \times$ very low overall quality

\section{Main conclusion (direct quote)}

"The body of evidence is insufficient to support conclusions about the effectiveness of vacuum-assisted closure in the treatment of wounds."

"Consequently, we agree with the conclusions of the previous technology assessment reports and systematic reviews $[29,55,57,60,63,64]$ that there is insufficient evidence to recommend the routine use of this technology."

"There are at present no results of adequate reliability which provide proof of the superiority of NPWT in comparison with conventional therapy and which would justify broad use of this method outside clinical trial settings." "There is a paucity of high-quality RCTs on TNP for wound management with sufficient sample size and adequate power to detect any differences between TNP and standard dressings.".

"Based on the evidence to date, the clinical effectiveness of NPWT to heal wounds is unclear."

$\sum^{*}$ Numbers refer to all primary studies included in the reviews.

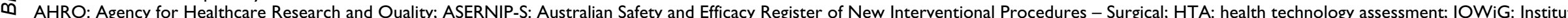

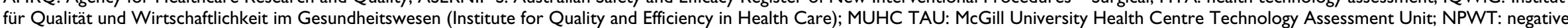
pressure wound therapy; OHTAC: Ontario Health Technology Advisory Committee; RCT: randomised controlled trial; TNP: topical negative pressure. 
bias [39], although it has been suggested that under certain conditions, estimates of effectiveness of non-RCTs may be valid if confounding is controlled for [40].

RCTs with adequately concealed allocation prevent selection bias and consequent distortions of treatment effects [41], and systematic reviews including RCTs represent the highest level of evidence for therapeutic interventions [42]. However, the quality and quantity of RCTs in surgical research is limited [43], and it has therefore been proposed not to base this type of research on RCTs alone $[36,44]$. Indeed, for some topics, non-RCTs are the only evidence available [45].

As for NPWT, although this treatment is widely applied in clinical practice, particularly in chronic wounds, at the time the IQWiG systematic review on NPWT was being planned only few RCTs were available; moreover, these were of poor quality [29]. However, there has been a recent increase in published RCTs, and as several of them are ongoing, more publications can be expected in the near future. One HTA agency has already changed its policy from including both RCTs and non-RCTs in systematic reviews on NPWT to one of including solely RCTs [32]. We agree with other researchers that non-RCTs should only be performed when RCTs are infeasible or unethical [38], and that systematic reviews including non-RCTs should only be conducted when RCTs are not available [39]. However, we emphasize that this should not be generalized to recommend excluding all kinds of non-randomised studies from systematic reviews on any topic and for any outcome of interest.

The type of non-RCT considered also differed: IQWiG's precondition for inclusion was the existence of a concurrent control group; studies with a historical control group were excluded, as systematic bias may arise from time trends in the outcomes of study participants [38].

Moreover, variations in the classification of study design were noted between reviews. For example, David Sampson, one of the other review authors, stated: "In general, our definition of randomized trials was probably more inclusive than yours. We decided to be inclusive due to the small number of potentially relevant studies available at that time. Our goal was to evaluate the quality of a larger pool of included studies rather than exclude more studies, based on quality concerns, to create a smaller pool of included studies" [personal communication].

As subjective factors are involved in the preparation of systematic reviews, inter-author variation is inevitable [46]. The evaluation of inter-author variation has shown that differences particularly affect the classification of study design $[46,47]$. One study showed that this was the case even when specific instructions and definitions were provided [47]. However, a recent analysis of the reproducibility of systematic reviews showed that, where authors were provided with guidelines for review preparation (including an algorithm to ensure that study designs were defined in a standardised manner), the overall reproducibility between reviews was good [48]. This finding emphasizes the relevance of standard reporting guidelines. The CONSORT statement on improving the quality of reporting for RCTs has been available for over a decade [49], and a revised version was published in 2001 [50]. In contrast, guidelines for non-RCTs are more recent $[51,52]$. The introduction of uniform reporting standards for nonRCTs may improve the future quality of reporting and lead to a closer agreement in the primary study citation and selection of systematic reviews.

Even though the reviews analysed included different numbers and types of studies, all reviews reached similar conclusions. This may be explained by the fact that the overall quality of the data on NPWT is poor.

\section{Conclusion}

The citation and selection of primary studies differ between systematic reviews on NPWT, primarily with regard to non-RCTs. These differences arise from variations in review methodology and inter-author classification of study design, as well as from different reporting styles for excluded studies. Uniform methodological and reporting standards need to be applied to ensure comparability between reviews as well as the validity of their conclusions.

\section{Competing interests}

The authors declare that they have no competing interests.

\section{Authors' contributions}

StL and FP initiated the study. FP coordinated the study and conducted the literature search. StL, FP, and StS screened and analysed the retrievals. NM and FP drafted the manuscript. All authors interpreted the data and made an intellectual contribution to the manuscript. All authors reviewed and approved the final version.

\section{Acknowledgements}

All work on this analysis was funded by IQWiG, an independent non-profit and non-government organisation that evaluates the quality and efficiency of health care services in Germany. IQWiG receives its commissions from the Federal Joint Committee (the decision-making body of the self-administration of the German health care services) as well as from the Federal Ministry of Health, and also undertakes projects and research work on its own initiative. All work on this study was supported by IQWiG within the framework of a systematic review on NPWT performed by IQWiG. Three authors (FP, NM, StL) are IQWiG full-time employees; one author (StS) works as an external expert for IQWiG. 


\section{References}

I. Hopayian $\mathrm{K}$ : The need for caution in interpreting high quality systematic reviews. BMJ 200I, 323:68I-684.

2. Jadad AR, Cook DJ, Browman GP: A guide to interpreting discordant systematic reviews. CMA] 1997, I 56:14||-1416 [http:// www.cmaj.ca/cgi/reprint/I56/10/141I?ck=nck].

3. Furlan AD, Clarke J, Esmail R, Sinclair S, Irvin E, Bombardier C: A critical review of reviews on the treatment of chronic low back pain. Spine 200I, 26:EI55-EI62.

4. Hoving JL, Gross AR, Gasner D, Kay T, Kennedy C, Hondras MA, Haines T, Bouter LM: A critical appraisal of review articles on the effectiveness of conservative treatment for neck pain. Spine 200I, 26:196-205.

5. Jadad AR, McQuay $H$ J: Meta-analyses to evaluate analgesic interventions: a systematic qualitative review of their methodology. J Clin Epidemiol 1996, 49:235-243.

6. Bland M, Rodgers M, Fayter D, Sowden A, Lewin R: Agreement in primary study selection between systematic reviews 2006 [http://wwwusers.york.ac.uk/ mb55/talks/meta agree.pdf]. York, UK, Centre for Reviews and Dissemination, University of York

7. Argenta LC, Morykwas MJ: Vacuum-assisted closure: a new method for wound control and treatment: clinical experience. Ann Plast Surg 1997, 38:563-577.

8. Negative pressure wound therapy. Final report (German version) 2006 [http://www.iqwig.de/download/N0403 Abschlussbericht Vakuumversiegelungstherapie zur Behandlung von Wunden..pdf]. Cologne, Germany, Institute for Quality and Efficiency in Health Care (IQWiG)

9. Negative pressure wound therapy. Rapid report (German version) 2007 [http://www.iqwig.de/download/N0602 Rapid Report Vakuumversiegelungstherapie von Wunden.pdf]. Cologne, Germany, Institute for Quality and Efficiency in Health Care (IQWiG)

10. Gregor S, Maegele M, Sauerland S, Krahn JF, Peinemann F, Lange S: Negative pressure wound therapy: a vacuum of evidence? Arch Surg 2008, 143:189-196.

II. Jüni P, Altman DG, Egger M: Assessing the quality of randomised controlled trials. In Systematic reviews in health care - meta-analysis in context Edited by: Egger M, Davey Smith G and Altman DG. London, UK, BMJ Publishing Group; 2001:87-108.

12. Higgins JPT GS: Cochrane Handbook for Systematic Reviews of Interventions 4.2.6 Updated September 2006. In The Cochrane Library Chichester, UK, John Wiley \& Sons, Ltd; 2006.

13. Catarino PA, Chamberlain MH, Wright NC, Black E, Campbell K, Robson D, Pillai RG: High-pressure suction drainage via a polyurethane foam in the management of poststernotomy mediastinitis. Ann Thorac Surg 2000, 70: |89|-|895.

14. Davydov IA, Abramov AI, Darichev AB: [Regulation of wound process by the method of vacuum therapy in middle-aged and aged patients]. Khirurgiia (Mosk) 1994:7-10.

15. Doss M, Martens S, Wood JP, Wolff JD, Baier C, Moritz A: Vacuumassisted suction drainage versus conventional treatment in the management of poststernotomy osteomyelitis. Eur J Cardiothorac Surg 2002, 22:934-938.

16. Eginton MT, Brown KR, Seabrook GR, Towne JB, Cambria RA: A prospective randomized evaluation of negative-pressure wound dressings for diabetic foot wounds. Ann Vasc Surg 2003, 1 7:645-649

17. Ford CN, Reinhard ER, Yeh D, Syrek D, De Las MA, Bergman SB, Williams S, Hamori CA: Interim analysis of a prospective, randomized trial of vacuum-assisted closure versus the healthpoint system in the management of pressure ulcers. Ann Plast Surg 2002, 49:55-6I.

18. Genecov DG, Schneider AM, Morykwas MJ, Parker D, White WL, Argenta LC: A controlled subatmospheric pressure dressing increases the rate of skin graft donor site reepithelialization. Ann Plast Surg 1998, 40:219-225.

19. Jeschke MG, Rose C, Angele P, Fuchtmeier B, Nerlich MN, Bolder U: Development of new reconstructive techniques: use of Integra in combination with fibrin glue and negative-pressure therapy for reconstruction of acute and chronic wounds. In Plast Reconstr Surg Volume I I 3. United States; 2004:525-530.

20. Joseph E, Hamori CA, Bergman S, Roaf E, Swann NF, Anastasi GW: A prospective randomized trial of vacuum-assisted closure versus standard therapy of chronic nonhealing wounds. Wounds A Compendium of Clinical Research and Practice 2000, 1 2:60-67.
21. Kamolz LP, Andel H, Haslik W, Winter W, Meissl G, Frey M: Use of subatmospheric pressure therapy to prevent burn wound progression in human: first experiences. In Burns Volume 30. England; 2004:253-258.

22. McCallon SK, Knight CA, Valiulus JP, Cunningham MW, McCulloch JM, Farinas LP: Vacuum-assisted closure versus saline-moistened gauze in the healing of postoperative diabetic foot wounds. Ostomy Wound Manage 2000, 46:28-34.

23. Moues CM, Vos MC, van den Bemd G], Stijnen T, Hovius SE: Bacterial load in relation to vacuum-assisted closure wound therapy: a prospective randomized trial. In Wound Repair Regen Volume 12. United States; 2004: I I- I7.

24. Scherer LA, Shiver S, Chang M, Meredith JW, Owings JT: The vacuum assisted closure device: a method of securing skin grafts and improving graft survival. Arch Surg 2002, I37:930-934.

25. Schrank C, Mayr M, Overesch M, Molnar J, Henkel von Donnersmarck G, Mühlbauer W, Ninkovic M: [Results of vacuum therapy (V.A.C.(R)) of superficial and deep dermal burns]. Zentralbl Chir 2004, I 29 Suppl I:S59-S6I.

26. Song DH, Wu LC, Lohman RF, Gottlieb LJ, Franczyk M: Vacuum assisted closure for the treatment of sternal wounds: the bridge between debridement and definitive closure. Plast Reconstr Surg 2003, I I I:92-97.

27. Wanner MB, Schwarzl F, Strub B, Zaech GA, Pierer G: Vacuumassisted wound closure for cheaper and more comfortable healing of pressure sores: a prospective study. Scand J Plast Reconstr Surg Hand Surg 2003, 37:28-33.

28. Wild T, Stremitzer S, Budzanowski A, Rinder H, TamandI D, Zeisel C, Holzenbein T, Sautner T: ["Abdominal Dressing" - A new method of treatment for open abdomen following secondary peritonitis]. Zentralbl Chir 2004, I 29 Suppl I:S20-S23.

29. Samson DJ, Lefevre F, Aronson N: Wound healing technologies: Lowlevel laser and vacuum-assisted closure 2004 [http://www.ahrq.gov/ downloads/pub/evidence/pdf/woundtech/woundtech.pdf]. Rockville, MD, USA, Agency for Healthcare Research and Quality (AHRQ)

30. Costa V, Brophy J, McGregor M: Vacuum-assisted wound closure therapy

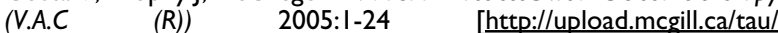
VAC REPORT FINAL.pdf]. Montreal, Quebec, Canada, McGill University Health Centre (MUHC) Technology Assessment Unit (TAU)

31. Pham CT, Middleton PF, Maddern GJ: The safety and efficacy of topical negative pressure in non-healing wounds: a systematic review. In J Wound Care Volume I5. England; 2006:240-250.

32. Ontario Health Technology Advisory Committee (OHTAC): Negative pressure wound therapy: Update 2006:I-38 [http://
[L www.health.gov.on.ca/english/providers/program/ohtac/tech/ reviews/pdf/rev npwt 070106.pdf]. Toronto, Ontario, Canada, Ministry of Health and Long-Term Care (MOHLTC) Medical Advisory Secretariat (MAS)

33. Egger M, Zellweger-Zahner T, Schneider M, Junker C, Lengeler $C$, Antes $G$ : Language bias in randomised controlled trials published in English and German. Lancet 1997, 350:326-329.

34. Moher D, Pham B, Lawson ML, Klassen TP: The inclusion of reports of randomised trials published in languages other than English in systematic reviews. Health Technol Assess 2003, 7:1-90.

35. Galandi D, Schwarzer G, Antes G: The demise of the randomised controlled trial: bibliometric study of the German-language health care literature, 1948 to 2004. BMC Med Res Methodol 2006, 6:30.

36. Gotzsche PC, Harden A: Searching for non-randomised studies 2000 [http://www.cochrane.dk/nrsmg/docs/chap3.pdf]. Kopenhagen, Dänemark, The Cochrane Non-Randomised Studies Methods Group (NRSMG) at The Nordic Cochrane Centre

37. Britton A, McKee M, Black N, McPherson K, Sanderson C, Bain C Choosing between randomised and non-randomised studies: a systematic review. Health Technol Assess 1998, 2:i-124 [http:// www.ncchta.org/fullmono/mon213.pdf].

38. Deeks JJ, Dinnes J, D'Amico R, Sowden AJ, Sakarovitch C, Song F, Petticrew M, Altman DG: Evaluating non-randomised intervention studies. Health Technol Assess 2003, 7:iii-I73.

39. Reeves BC, van Binsbergen J, van Weel C: Systematic reviews incorporating evidence from nonrandomized study designs: reasons for caution when estimating health effects. Eur J Clin Nutr 2005, 59 Suppl I:SI55-SI6I.

40. MacLehose RR, Reeves BC, Harvey IM, Sheldon TA, Russell IT, Black AM: A systematic review of comparisons of effect sizes 
derived from randomised and non-randomised studies. Health Technol Assess 2000, 4:I-I54 [http://www.hta.ac.uk/fullmonol mon434.pdf].

4I. Kunz R, Vist G, Oxman AD: Randomisation to protect against selection bias in healthcare trials [online] 2002 [http://www.mrw.inter science.wiley.com/cochrane/clmethrev/articles/MR0000l2/pdf fs.html]. Chichester, UK, John Wiley \& Sons, Ltd

42. Levels of evidence and grades of recommendation 200I [http://] www.cebm.net/levels of evidence.asp]. Oxford, UK, Centre for Evidence-Based Medicine (CEBM)

43. McCulloch P, Taylor I, Sasako M, Lovett B, Griffin D: Randomised trials in surgery: problems and possible solutions. $B M] 2002$ 324:|448-I45I [http://bmi.bmijournals.com/cgi/reprint/324/735I/ 1448].

44. Guller U: Surgical outcomes research based on administrative data: inferior or complementary to prospective randomized clinical trials? World J Surg 2006, 30:255-266.

45. Fraser C, Murray A, Burr J: Identifying observational studies of surgical interventions in MEDLINE and EMBASE. BMC Med Res Methodol 2006, 6:4I [http://www.biomedcentral.com//47|-2288/ 6/4I].

46. Strang WN, Boissel P, Uberla K: Inter-reader variation. The Cochrane Methodology Register 1997 [http://www.mrw.interscience.wiley.com/ cochrane/clcmr/articles/CMR-660/frame.html]. West Sussex, United Kingdom, John Wiley \& Sons Ltd

47. Bombardier C, Jadad A, Tomlinson G: What is the study design? The Cochrane Methodology Register 2004 [http://www.mrw.inter science.wiley.com/cochrane/clcmr/articles/CMR-6639/frame.html].

West Sussex, United Kingdom, John Wiley \& Sons Ltd

48. Thompson R, Bandera E, Burley V, Cade J, Forman D, Freudenheim J, Greenwood D, Jacobs D, Kalliecharan R, Kushi L, McCullough M, Miles L, Moore D, Moreton J, Rastogi T, Wiseman M: Reproducibility of systematic literature reviews on food, nutrition, physical activity and endometrial cancer. Public Health Nutr 2007: I-9.

49. Begg C, Cho M, Eastwood S, Horton R, Moher D, Olkin I, Pitkin R, Rennie D, Schulz KF, Simel D, Stroup DF: Improving the quality of reporting of randomized controlled trials. The CONSORT statement. JAMA 1996, 276:637-639.

50. Moher D, Schulz KF, Altman D: The CONSORT statement: revised recommendations for improving the quality of reports of parallel-group randomized trials. JAMA 200I, 285: | 987-199| [http://jama.ama-assn.org/cgi/reprint/285//5//987].

5I. Des Jarlais DC, Lyles C, Crepaz N: Improving the reporting quality of nonrandomized evaluations of behavioral and public health interventions: the TREND statement. Am J Public Health 2004, 94:36I-366 [http://www.trend-statement.org/asp/documents/ statements/AIPH Mar2004 Trendstatement.pdf

52. von Elm E, Altman DG, Egger M, Pocock SJ, Gotzsche PC, Vandenbroucke JP: The Strengthening the Reporting of Observational Studies in Epidemiology (STROBE) statement: guidelines for reporting observational studies. Ann Intern Med 2007, 147:573-577 [http://www.annals.org/cgi/reprint//47/8/ 573 dff

53. Andros G, Armstrong DG, Attinger CE, Boulton AJ, Frykberg RG, Joseph WS, Lavery LA, Morbach S, Niezgoda JA, Toursarkissian B: Consensus statement on negative pressure wound therapy (V.A.C. Therapy) for the management of diabetic foot wounds. Ostomy Wound Manage 2006, Suppl: I-32.

54. Brem H, Sheehan P, Rosenberg HJ, Schneider JS, Boulton AJ: Evidence-based protocol for diabetic foot ulcers. Plast Reconstr Surg 2006, II 7:193S-209S.

55. Ubbink DT, Westerbos SJ, Evans D, Land L, Vermeulen H: Topical negative pressure for treating chronic wounds. The Cochrane Database of Systematic Reviews 200I:CD001898 [http:// www.mrw.interscience.wiley.com/cochrane/clsysrev/articles/ CD00 1898/pdf fs.html]. West Sussex, United Kingdom, John Wiley \& Sons Ltd

56. Fleck T, Gustafsson R, Harding K, Ingemansson R, Lirtzman MD, Meites HL, Moidl R, Price P, Ritchie A, Salazar J, Sjogren J, Song DH, Sumpio BE, Toursarkissian B, Waldenberger F, Wetzel-Roth W: The management of deep sternal wound infections using vacuum assisted closure (V.A.C.) therapy. Int Wound J 2006, 3:273-280.

57. Fisher A, Brady B: Vacuum assisted wound closure therapy 2003 [http:// cadth.ca/media/pdf/22I vac cetap e.pdf]. Ottawa, Ontario, Canada, The Canadian Coordinating Office for Health Technology Assessment (CCOHTA)
58. Gray M, Peirce B: Is negative pressure wound therapy effective for the management of chronic wounds? J Wound Ostomy Continence Nurs 2004, 3 I: I0I- 105.

59. Inc $\mathrm{H}$ : Negative pressure wound therapy for wound healing Lansdale, Pennsylvania, USA, Hayes, Inc; 2003.

60. Higgins S: The effectiveness of vacuum assisted closure (VAC) in wound healing 2003 [http://www.mihsr.monash.org/cce/res/pdf/c/99lfr.pdf]. Clayton, Australia, Centre for Clinical Effectiveness (CCE)

61. Mayer ED, Boukamp K, Simoes E: Vakuumversiegelung in der Wundbehandlung - Verfahren nach EbM-Kriterien evaluiert? Bewertung aus sozialmedizinischer Sicht Lahr (Schwarzwald), Medizinischer Dienst der Krankenkassen (MDK) Baden-Württemberg; 2002.

62. Mendonca DA, Papini R, Price PE: Negative-pressure wound therapy: a snapshot of the evidence. Int Wound J 2006 , 3:26I-27I.

63. Ontario Health Technology Advisory Committee (OHTAC): Vacuum assisted closure therapy for wound care 2004:I-59 [http:/l www.health.gov.on.ca/english/providers/program/ohtac/tech/ techlist 2004.html]. Toronto, Ontario, Canada, Ministry of Health and Long-Term Care (MOHLTC) Medical Advisory Secretariat (MAS)

64. Pham C, Middleton P, Maddern G: Vacuum-assisted closure for the management of wounds: an accelerated systematic review 2003 [http:w.surs.org/AM/Template.cfm?Sec tion=ASERNIP S Publications\&Template $=/ \mathrm{CM} /$ Content Disay.cfm\&ContentFilelD=|49|]. Adelaide, Australia, Australian safety and efficacy register of new interventional procedures - surgical (ASERNIP-S)

65. Shirakawa M, Isseroff RR: Topical negative pressure devices: use for enhancement of healing chronic wounds. Arch Dermatol 2005, I 41: : 1449-1453.

66. Suess J], Kim PJ, Steinberg JS: Negative pressure wound therapy: evidence-based treatment for complex diabetic foot wounds. Curr Diab Rep 2006, 6:446-450.

67. Turina M, Cheadle WG: Management of established surgical site infections. Surgical Infections 2006, 7 (Suppl 3):S33-S4I.

68. Ubbink DT, Vermeulen H, Lubbers M]: [Local wound care: evidence-based treatments and dressings]. Ned Tijdschr Geneeskd 2006, I50: II65-1172.

69. Whelan C, Stewart J, Schwartz BF: Mechanics of wound healing and importance of Vacuum Assisted Closure in urology. Urol 2005, 173:1463-1470.

70. Costa V, Brophy J, McGregor M: Vacuum-assisted wound closure therapy $\begin{array}{llll}\text { (V.A.C } & \text { (R)) 2007:I-24 [http://upload.mcgill.ca/taul }\end{array}$ VAC REPORT FINAL.pdf]. Montreal, Quebec, Canada, McGill University Health Centre (MUHC) Technology Assessment Unit (TAU)

\section{Pre-publication history}

The pre-publication history for this paper can be accessed here:

http://www.biomedcentral.com/1471-2288/8/41/prepub

Publish with BioMed Central and every scientist can read your work free of charge

"BioMed Central will be the most significant development for disseminating the results of biomedical research in our lifetime. "

Sir Paul Nurse, Cancer Research UK

Your research papers will be:

- available free of charge to the entire biomedical community

- peer reviewed and published immediately upon acceptance

- cited in PubMed and archived on PubMed Central

- yours - you keep the copyright
BioMedcentral 